\title{
Ein Batterie-Verstärker für Zählrohre mit betriebssicherer Erzeugung der Zählrohrspannung aus einer Akkumulatoren-Batterie
}

\author{
Von ARnold Flammersfeld
}

\author{
Aus dem Kaiser-Wilhelm-Institut für Chemie, Tailfingen \\ (Z. Naturforschg. 1, 168-170 [1946]; eingegangen am 20. Februar 1946)
}

Z ahlreiche Stadtnetze werden jetzt zeitweise und unregelmäßig abgeschaltet, so daß die üblichen Netzverstärker für Zählrohre nicht dauernd betriebsfähig sind. Für die Messung von schwachen, langlebigen Präparaten ist dies in vielfacher Hinsicht äußerst lästig. Es wurden daher bei uns Batterieverstärker gebaut, die sich im Dauerbetrieb sehr bewährt haben, und die sogar für gewisse sich über Monate hinziehende Messungen den Netzverstärkern eindeutig überlegen sind, auch wenn das Netz nicht abgeschaltet würde. Sie werden von den sich über das Netz verbreitenden Störungen viel weniger beeinflußt und geben deshalb z. B. eine vorzügliche Konstanz des Nulleffekts. Es ist daher vielleicht erwünscht, die Anordnung kurz zu beschreiben.

Die Hauptschwierigkeit bei einem Batterieverstärker ist die Erzeugung der 1000 bis 2000 Volt einstellbarer Hochspannung für das Zählrohr. Sie wird hier in neuartiger Weise gelöst, indem mit Hilfe zweier Elektronenröhren (in praxi eine sog. Verbundröhre) aus dem Batteriegleichstrom ein Hackstrom gewonnen wird, der an Stelle von Wechselstrom durch einen gewöhnlichen Transformator fließt. Im Prinzip sollte das auch mit einem mechanischen Zerhacker möglich sein; da derartige Instrumente aber nicht selbst betriebssicher herzustellen sind, wurde diese Röhrenanordnung gewählt, die seit mehreren Monaten störungsfrei arbeitet und den Vorzug besitzt, praktisch geräuschlos zu sein.

Der Aufbau der Anordnung ist folgender: der Verstärkerteil besteht aus drei Batterieröhren. Der Hochspannungsteil besteht aus einer Doppelröhre VCL 11, deren Anodenstrom einen Transformator mit Gleichrichter speist, und einem Stabilisator nach Medicus zur Konstanthaltung der Zählspannung. Alles zusammen ist selbstverständlich in einem Gehäuse untergebracht und benötigt zum Betrieb einen Heizakkumulator, eine Gittervorspannungsbatterie $30 \mathrm{~V}$ und einen Anschluß an eine 120-V-Akkumulatorbatterie (Hausbatterie). Das genaue Schaltschema zeigt Abb.1. Der Nachbau wird danach ohne weiteres möglich sein. Durch Verändern einiger weniger Schaltelemente kann er natürlich für andere Batteriespannungen, z. B. $200 \mathrm{~V}$, angepaßt werden. Im einzelnen muß auf folgendes hingewiesen werden:

1. Verstärkerteil. Der Verstärkerteil besteht aus 3 Röhren, von denen die erste (KF 4) als NeherHarper-Röhre ${ }^{1}$ wirkt. Die von deren Anode (Zählspannung) abgenommenen negativen Impulse werden in den beiden weiteren Röhren KC 1 und KL 1 verstärkt und dem Zählwerk zugeführt. Die beiden letzten Röhren sind zu einem Kippgenerator verbunden, welcher aber wegen großer Gitterspannung der letzten Röhre nur auf jedesmaligem äußeren Anstoß von der KF 4 einen Stromstoß liefert. Mit Hilfe dieser Schaltung erhält man von dem Zählstoß einen genau einstellbaren Stromimpuls konstanter Länge für das Zählwerk. Gegenüber einer von $\mathrm{Neher}^{2}$ angegebenen ähnlichen Schaltung stellt die hier gebrauchte eine wesentliche Verbesserung dar. In der Ne her schen Schaltung, in der die Gegenkopplung (Kondensator $C_{2}$ ) von der Anode ausgeht, stören die infolge der Bewegung des Ankers in den Zählwerkspulen auftretenden Spannungen sehr stark, da sie sich vermöge dieses Kondensators auf das Gitter der zwei-

1 H. V. Neher u. W. H. Harper, Physic. Rev. 49, 940 [1936].

2 H. V. Neher, Rev. Sci. Instruments 10, 29 [1939]. 
ten Röhre übertragen und dort verstärkt zurückkommen. So werden bei größerer Teilchenzahl Form und Intensität der Zählwerkimpulse sehr unregelmäßig, und der Vorteil einer solchen Anordnung geht gerade bei großen Teilchenzahlen verloren. Von diesem Fehler ist die vorliegende Schaltung weitgehend frei, da die Gegenkopplung vom
Netzverstärkern seit langer Zeit ausgezeichnet bewährt. Je nach der Steilheit der Röhren müssen die Kopplungsglieder geringfügig verändert werden, um gutes Arbeiten zu erzielen.

2. Hochspannungsteil. Die Hochspannung wird erzeugt von einem Hochspannungstransformator, durch dessen Primärwicklung der Anodenstrom

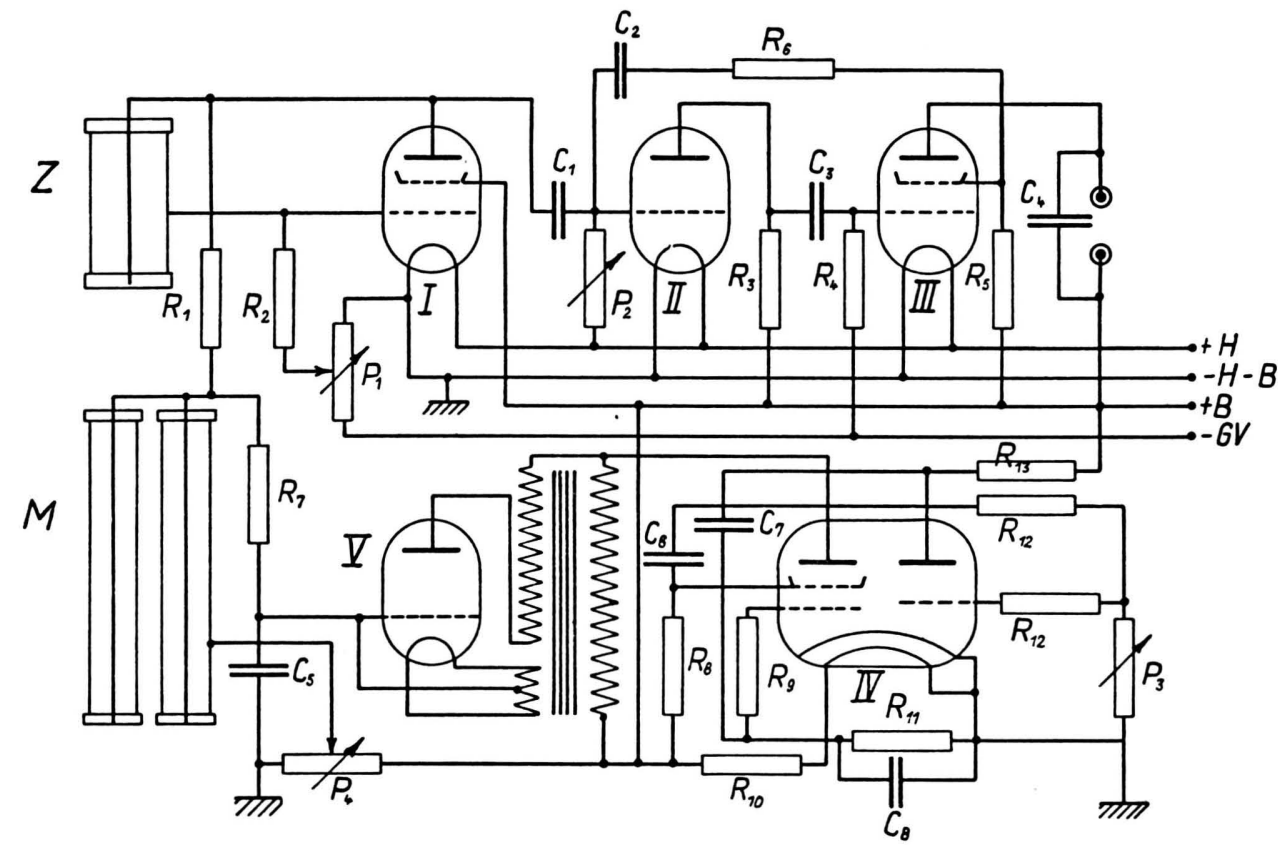

Abb. 1. Schaltschema. Stückliste:

$$
\text { Wi derstände: }
$$

$\begin{array}{llllllll}R_{1} & 100 \mathrm{M} \Omega & R_{4} & 500 \mathrm{k} \Omega & R_{7} & 100 \mathrm{M} \Omega & R_{10} & 600\end{array}$ $\begin{array}{lllllllll}R_{2} & 40 \mathrm{M} \Omega & R_{5} & 50 \mathrm{k} \Omega & R_{8} & 5 \mathrm{k} \Omega & R_{11} & 50 \mathrm{k} \Omega\end{array}$

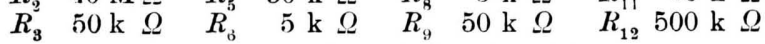
$R_{13} 5 \mathrm{k} \Omega \quad \mathrm{R}_{14} 5 \mathrm{k} \Omega$ (neben $\mathrm{R}_{13}$; in der Zeichnung irrtümlich $\mathrm{R}_{12}$ )

Schirmgitter ausgeht, welches durch die beträchtlichen Spannungsschwankungen an der Anode gar nicht beeinflußt wird. Dementsprechend sind auch bei den höchsten Teilchenzahlen die Impulse alle gleich groß, was einwandfreies Arbeiten des Zählwerkes verbürgt. Auch die Einstellbarkeit der Impulslänge am Potentiometer $P_{2}$ ist für die Erzielung eines optimalen Auflösungsvermögens ein großer Vorteil. Diese Schaltung für Impulse konstanter Länge kann natürlich mit allen Arten von Röhren realisiert werden und hat sich bei uns auch bei den

$$
\begin{aligned}
& \begin{array}{l}
\text { Potentiometer: } \\
P_{1} 1 \mathrm{M} \Omega \operatorname{lin} \quad P_{2} 1 \mathrm{M} \Omega \log \quad P_{3} 1 \mathrm{M} \Omega \log P_{4} 500 \mathrm{k} \Omega \operatorname{lin}
\end{array} \\
& \text { Kondensatoren: } \\
& \begin{array}{cccc}
\multicolumn{4}{c}{C_{1} \text { ca. } 30 \mathrm{pF} 2000 \mathrm{~V} \text { Betriebssp. }} \\
C_{2} 5000 \mathrm{pF} & C_{4} 0.1 \mu \mathrm{F} & C_{8} 5000 \mathrm{pF} \\
C_{3} 50000 \mathrm{pF} & C_{5} \text { ca. } 0.1 \mu \mathrm{F} 2000 \mathrm{~V} & C_{7} 5000 \mathrm{pF} \\
& & \mathrm{C}_{8} 2000 \mathrm{pF} &
\end{array} \\
& \text { Röhren: } \\
& \text { I KF } 4 \text { II KC } 1 \text { III KL } 1 \text { IV VCL } 11 \quad \text { V KC } 1
\end{aligned}
$$

einer VCL 11 fließt, deren Trioden- und Tetrodenteil in ähnlicher Weise zu einem Kippgenerator verbunden sind wie die KC $1+$ KL 1 des Verstärkerteils. Hier sind aber die Gittervorspannungen beider Einzelsystemteile Null, so daß die Röhren dauernd schwingen. Zum besseren Verständnis muß auf einiges Ungewöhnliche in dieser Betriebsart hingewiesen werden. Der Transformator ist ein gewöhnlicher Netztransformator für primär $220 \mathrm{~V}$ und sekundär $2000 \mathrm{~V}$ effektiv, $10 \mathrm{~mA}$, mit einer Gleichrichterwicklung $4 \mathrm{~V}, 1 \mathrm{~A}$, wie er gewöhnlich 
in netzbetriebenen Zählrohrspannungsgeräten benutzt wird. Der Anodenstrom der Tetrode der VCL 11, der mit $120 \mathrm{~V}$ Anodenspannung erzielt wird, reicht natürlich nicht aus, den Transformator voll zu erregen. Aber da die Kurvenform des Anodenstroms keine sinusförmige, sondern eine rechteckige ist, gibt dieser Strom nach der Transformation in der Sekundärspule sehr starke Spannungsspitzen (ähnlich wie beim Funkeninduktor), auch wenn der quadratische Mittelwert der Spannung längst nicht $2000 \mathrm{~V}$ beträgt. So kommt man nach Gleichrichtung der richtigen Phase (ausprobieren!) mühelos auf etwa $2000 \mathrm{~V}$ Gleichspannung, die vollkommen ausreichend sind. Als Gleichrichterröhre wird eine $\mathrm{KC} 1$ benutzt, die nur geringe Heizleistung benötigt $(2 \mathrm{~V}, 65 \mathrm{~mA})$ und alle sonstigen Beanspruchungen aushält. Sie wird an die 4-V-Wicklung angeschlossen, doch müssen beim Inbetriebsetzen die Spannungen vorher gemessen und nötigenfalls mit dem Potentiometer $P_{3}$ die optimalen Bedingungen aufgesucht werden (Frequenz etwa 200-400 Schwingungen pro Sekunde).

Zur Erzeugung einer konstanten, grob einstellbaren und in gewissen Grenzen fein regulierbaren Zählrohrspannung kann hier nicht, wie sonst bei unseren Verstärkern üblich, eine Elektronenröhren-Stabilisierung verwendet werden, da der Hochspannungsteil hierzu nicht leistungsfähig genug ist. Es wird daher ein Stabilisator nach Medicu s ${ }^{3}$ verwendet, der mit in das Gehäuse eingebaut wird.

\footnotetext{
${ }^{3}$ H. Medicus, Z. techn. Physik 14, 304 [1933].
}

Er besteht in diesem Falle aus zwei Messingröhren $M$ von $15 \mathrm{~mm}$ Durchmesser und $350 \mathrm{~mm}$ Länge (Draht 0,2 $\mathrm{mm}$ Durchmesser), die elektrisch und pneumatisch parallel geschaltet sind. Zur Grobeinstellung der Spannung muß der Gasdruck variiert werden, was mit einer Wasserstrahlpumpe mühelos möglich ist, da der benötigte Druck zwischen 20 und $80 \mathrm{~mm} \mathrm{Hg}$ liegt. Zur Feineinstellung wird folgender Kunstgriff benutzt: der Minuspol des Stabilisators (Gehäuse) ist nicht mit der Erde, sondern mit einem Potentiometer $P_{4}$ verbunden, das beliebige Spannungen zwischen Null und der vollen Batteriespannung abzugreifen gestattet. Damit hat man eine Drehknopfeinstellung der Zählerspannung, die zur genauen Einstellung des Arbeitspunktes und der Kontrolle der Einsatzspannung vollkommen ausreicht.

Es braucht wohl nicht extra betont zu werden, daß die angegebenen Röhren und Schaltungselemente nicht kritisch sind. Selbstverständlich kann man beliebige andere Röhren verwenden, deren Heizung ökonomisch ist, z. B. Röhren der E-, V-oder U-Serie. Auch als erste Röhre der Kippgeneratoren ist sowohl eine Triode wie eine Pentode mit gleichem Erfolge $\mathrm{zu}$ verwenden. Insbesondere könnte man in dem angegebenen Gerät an Stelle der VCL 11 des Hochspannungsteiles zwei Batterieröhren verwenden und diese aus dem Heizakkumulator speisen. Dann könnte der zum Betrieb des Transformators nötige Strom und die übrigen Anodenströme (zusammen höchstens $10 \mathrm{~mA}$ ) einer gewöhnlichen Anodenbatterie entnommen werden, wodurch das Gerät tragbar wird. 